\title{
Activités
}

18-1 | 2021

Le programme de recherche cours d'action (2)

\section{Apprendre à déceler le potentiel de développement des situations de travail : l'exemple de conseillers agricoles face aux enjeux de l'agro-écologie}

Learning to detect the developmental potential of work situations: the case of agricultural advisors facing agro-ecological challenges

\section{Sophie Duhamel, Paul Olry et Marianne Cerf}

\section{OpenEdition \\ Journals}

Édition électronique

URL : http://journals.openedition.org/activites/5983

DOI : 10.4000/activites.5983

ISSN : 1765-2723

Éditeur

ARPACT - Association Recherches et Pratiques sur les ACTivités

\section{Référence électronique}

Sophie Duhamel, Paul Olry et Marianne Cerf, « Apprendre à déceler le potentiel de développement des situations de travail : l'exemple de conseillers agricoles face aux enjeux de l'agro-écologie », Activités [En ligne], 18-1 | 2021, mis en ligne le 15 avril 2021, consulté le 17 avril 2021. URL : http:// journals.openedition.org/activites/5983; DOI : https://doi.org/10.4000/activites.5983

Ce document a été généré automatiquement le 17 avril 2021

\section{cc) (1)}

Activités est mis à disposition selon les termes de la licence Creative Commons Attribution - Pas d'Utilisation Commerciale - Pas de Modification 4.0 International. 


\section{Apprendre à déceler le potentiel de développement des situations de travail : l'exemple de conseillers agricoles face aux enjeux de l'agro-écologie}

Learning to detect the developmental potential of work situations: the case of agricultural advisors facing agro-ecological challenges

Sophie Duhamel, Paul Olry et Marianne Cerf

\section{NOTE DE L'ÉDITEUR}

Article soumis le 30 septembre 2020, accepté le 23 février 2021

Remerciements : À l'ensemble des participant.e.s projet Cas-Dar CHANGER et plus particulièrement à celles et ceux du dispositif qui a été analysé dans cet article. Ce travail a bénéficié du financement du Cas-Dar « innovation et partenariat » du Ministère de l'Agriculture, de l'Alimentation et de la Forêt dans le cadre du projet CHANGER.

\section{Introduction}

1 La mise en exergue de l'importance d'axer la formation professionnelle sur les situations de travail a fait l'objet de nombreux travaux visant à montrer l'efficacité d'une mise en situation pour accroître les compétences des individus (Barbier, Berton, \& Boru, 1995 ; Duhamel, Cerf, \& Olry, 2017 ; Mayen \& Olry, 2012 ; Pastré, 2011 ; Pastré, Mayen, \& Vergnaud, 2006). Comme le disent Mayen \& Olry (2012) « le concept de situation est un concept fondateur en didactique professionnelle [...] qui désigne ce avec quoi une 
personne est amenée à agir ». S'il est classique, dans une perspective de formation professionnelle, de considérer que les situations de travail sont "à apprendre", la montée en puissance de l'AFEST ${ }^{1}$ met en avant qu'elles sont aussi le moyen d'apprentissages et de développement, puisqu'on peut "apprendre d'elles». Les situations de travail possèdent des configurations physique, institutionnelle, technique et sociale dans et avec lesquelles un professionnel est amené à agir. C'est une forme sociale, une unité relativement circonscrite, faite de propriétés relativement stables et identifiables, mais susceptible d'évolutions. Sous certaines conditions, qu'il s'agit alors de préciser, elles sont susceptibles de favoriser des apprentissages et un développement de l'activité du sujet (Mayen, 2012).

Dans le projet technologique de la didactique professionnelle (Mayen, 2013), une question centrale est d'identifier i) si quelque chose peut s'apprendre en situation, ii) si celle-ci détient un potentiel d'aménagement pour le faire apprendre, iii) si un intervenant peut réaliser ce potentiel en créant des conditions d'apprentissage soutenant un développement professionnel. Nous avons eu l'opportunité d'observer un dispositif mis en place auprès de professionnels ${ }^{2}$ qui agissent avec et pour autrui et dont les activités et situations évoluent tout en n'étant pas (encore) stabilisées. Un des enjeux est, pour eux, comme pour les bénéficiaires de leur action, de reconstruire ensemble le sens des situations et des activités réalisées. Ces professionnels, des conseillers agricoles, sont en cours de reconfiguration de leurs activités pour accompagner des agriculteurs dans une évolution de leurs pratiques afin de répondre à des problématiques environnementales (réduction des pollutions dans les eaux, des effets négatifs sur la biodiversité, atténuation du, et adaptation au, changement climatique...). Le dispositif a été construit pour aider ces professionnels, individuellement et collectivement, à analyser le couplage qui s'opère dans l'action entre le sujet et la situation pour y agir efficacement. Dit autrement, il s'agit, de permettre une analyse de l'activité du point de vue des ajustements opérés entre ce que fait un professionnel, ce qui oriente ce qu'il fait, et les dimensions agissantes d'une situation. Ces dernières sont définies par (Mayen, Métral, \& Tourmen, 2010, p. 34) comme les "variables essentielles à prendre en compte dans l'activité ».

Dans ce qui suit, nous évoquons le contexte professionnel dans lequel s'inscrit notre étude, à savoir les enjeux d'évolution du métier de conseiller agricole pour accompagner les agriculteurs vers l'agro-écologie. Nous exposons ensuite le dispositif qui a été conçu pour aider des conseillers à faire face à ces enjeux en pointant les bases conceptuelles issues de la didactique professionnelle sur lesquelles s'ancre ce dispositif. Nous présentons ensuite les données qui sont mobilisées dans ce texte, ainsi que leur analyse, pour pointer comment, dans ce dispositif, les conseillers redécouvrent des situations de travail connues et s'approprient, de façon différenciée, le potentiel de développement de ces situations.

\section{Des professionnels du conseil agricole face à la triple extension des situations sociales de conseil}

4 Les conseillers agricoles entretiennent et exercent une relation de service avec les agriculteurs c'est-à-dire, en s'inspirant de la définition donnée par Cerf et Falzon (2005) une relation asymétrique entre un professionnel (ici, le conseiller) et un nonprofessionnel (ici l'agriculteur) ce dernier formulant une demande et identifiant qu'il n'est pas à 
même de la satisfaire sans avoir recours aux moyens que le professionnel met à sa disposition tout en gardant, au moins pour partie, la propriété de ces moyens. Dans ce cadre, conseiller et agriculteur sont en situation de complémentarité : il est impossible à chacun d'atteindre seul le résultat qui constituera le service. Classiquement dans le conseil agricole en production végétale, cela signifie que le conseiller apporte des connaissances scientifiques et techniques et se repose sur des références techniques fondées sur des expérimentations scientifiques et techniques menées au champ, sur son expertise acquise au contact des agriculteurs et des collègues, et via ses lectures. L'agriculteur quant à lui apporte un cas pratique et une expérience située, sa parcelle. Dans la situation sociale de conseil classique, les agriculteurs sont en attente d'une réponse technique efficiente face à un problème ponctuel apparaissant dans la conduite d'une culture sur cette parcelle à un temps donné. Ainsi, l'agriculteur exprime un problème et le conseiller se doit d'en identifier la cause et de proposer, de prescrire, une solution adéquate. Dans cette situation sociale, l'expertise du conseiller est recherchée, mise en valeur et en avant.

5 Aujourd'hui, les conseillers sont confrontés à une double injonction: (i) celle des pouvoirs publics et de la société qui demandent une transition vers des pratiques plus agro-écologiques et (ii) celle venant des agriculteurs qui peuvent être, pour certains, prêts à explorer des solutions inédites, pour d'autres, réticents ou démunis pour engager un tel changement de pratiques. De ce fait, ils doivent opérer « un certain renversement des perspectives»: il s'agit pour eux «moins d'apporter des réponses qu'aider à formuler les bonnes questions, celles qui permettent aux agriculteurs d'y voir plus clair et d'avancer" (Lemery, 2003, p.6.). Cela invite à mobiliser les capacités de conception des agriculteurs comme certains auteurs les repèrent au cours de transitions vers des pratiques plus agro-écologiques (Chantre, 2011 ; Chizallet, Prost, \& Barcellini, 2020 ; Coquil, 2014 ; Prost, Berthet, Cerf, Jeuffroy, Labatut, \& Meynard, 2017,). Pour le conseiller, habitué à considérer l'agriculteur comme un décideur mais plus rarement comme un concepteur, s'ouvre alors une interrogation sur ce que peuvent être "ces bonnes questions" évoquées par Lemery (ibid.) et la façon de conduire l'agriculteur à les formuler dans une perspective de reconception de son système de travail, tout en l'aidant aussi à mettre en œuvre dans la durée les solutions qu'il envisage.

6 Ces éléments permettent de pointer que les situations de travail coopératif entre ces deux professionnels évoluent. Néanmoins, les nouvelles situations restent peu définies en termes de contenu échangé et de modalités de réalisation. Gagneur (2011), dans son travail de recherche sur la modélisation de la relation de conseil, met en avant une triple extension de la situation socio-agro-écologique.

"L'extension cognitive par l'incorporation d'objets et de dimensions nouvelles appelant de nouveaux champs de connaissances (p. e. migration des résidus de produits phytosanitaires dans les nappes phréatiques); l'extension sociale par l'incorporation d'acteurs nouveaux amenant leurs préoccupations dans le champ professionnel agricole (p.e. Agences de l'eau); et l'extension politique car la conjonction des deux points précédents [qui] remet en question la construction d'objectifs politiques partagés au sein des organisations pourvoyeuses de conseil» (p. 7).

Cette triple extension des situations sociales de conseil induit pour les conseillers des changements à opérer dans le couplage entre le professionnel et la situation non seulement pour agir, mais aussi pour repérer ces extensions et leurs conséquences sur l'action. Les conseillers agricoles doivent alors développer de nouveaux modes de pensée, car ces extensions modifient "profondément les exigences cognitives» (Mayen, 
2014, p. 38) pour agir en situation. Différents projets ont été mis en œuvre pour accompagner les conseillers (Delbos, David, Minas, Cerf, Falgas, Gagneur et al., 2014 ; Guillot, Cerf, Petit, Olry, \& Omon, 2013 ; Omon, Cerf, Auricoste, Olry, Petit, \& Duhamel, 2019) considérant plus ou moins explicitement que la relation de conseil devait évoluer vers une relation de coproduction du conseil avec l'agriculteur (Cerf \& Maxime, 2006). Le conseiller devrait ainsi permettre à l'agriculteur de se construire une nouvelle place d'acteur et concepteur de son système de travail, tout en se disposant à coproduire le conseil.

\section{Un dispositif qui met en travail le couplage entre la situation sociale de conseil et le conseiller}

7 Le dispositif que nous avons étudié s'est inscrit dans le projet Changer (Omon et al., 2019), financé par le Compte d'Affectation Spécialisé pour le Développement Agricole et Rural dans le cadre du programme "innovation et partenariat» du Ministère de l'Agriculture. Ce projet vise «la professionnalisation des agents face à de nouvelles formes d'accompagnement des agriculteurs dans le changement en productions végétales à travers une dynamique de réseau d'échanges sur le métier » (document du projet, 2014). Pour ce faire, ont été mis en place une dizaine de dispositifs d'animation d'échanges entre des conseillers, dont celui sur lequel s'appuient les résultats que nous présentons. Ces dispositifs sont construits pour échanger sur des situations de conseil rencontrées par les participants durant trois ans.

\subsection{Les bases conceptuelles sur lesquelles se fonde le dispositif}

8 Mayen (2010) précise que "les situations sont toujours des situations sociales" possédant un potentiel de développement de par les relations sociales qui s'y déroulent et les rôles des acteurs au sein de la situation. Mayen (ibid.) propose ainsi le concept de situation potentielle de développement (SPD) en s'inspirant des travaux de Vygotski (1988) sur la situation sociale de développement vue comme «le point de départ de tous les changements dynamiques qui se produisent dans le développement pendant une période donnée " (Mayen \& Orly, 2012, p. 94). Ce concept, ou celui de potentiel de développement d'une situation (Gagneur, 2010; Gagneur \& Mayen, 2010), désigne ce par quoi les caractéristiques d'une situation donnée peuvent engendrer (ou pas) des processus d'apprentissage et de développement, lorsqu'elles entrent dans le champ de l'activité d'un professionnel ou futur professionnel. Ainsi, pour Mayen et Olry (2012), les situations potentielles de développement sont « cette conjonction entre d'une part, les conditions externes qui, comme l'écrit Vygotski (1985), «déterminent les modalités concrètes selon lesquelles la période critique se manifeste puis disparaît » (p. 94) et, d'autre part, la logique interne de la dynamique de la vie professionnelle (ou sociale) d'une personne (ses motifs, ses capacités) à une période donnée.

9 En ce sens, une situation de travail est vue comme une situation potentielle de développement pour l'individu à condition que cette situation rassemble "l'ensemble des conditions pour engager puis étayer le processus de développement des compétences d'un individu» (Mayen, 1999, p. 66). Dans cette perspective la situation de travail est un élément clef «pour engager et générer " un processus de développement professionnel (Mayen, 2007) qui doit favoriser la construction de nouveaux couplages entre un sujet 
et une (des) situation(s) ou, dit autrement, l'activité et les actions s'y rattachant. Si un potentiel de développement pour l'individu et la situation émerge, il est alors possible, pour un intervenant, d'amorcer un processus de didactisation des situations professionnelles pour les rendre apprenantes. Selon Pastré (2011), ce processus repose sur ce qu'il appelle "une structure conceptuelle de la situation", matrice commune à des situations similaires et mobilisant des conceptualisations proches par celles et ceux qui y agissent. Néanmoins dans les situations de service, cette proposition s'avère peu efficiente (Mayen \& Thievenaz, 2018), et ce d'autant moins qu'il n'est pas évident de savoir ce qu'il convient de faire dans la situation professionnelle considérée pour atteindre les buts de l'action. L'enjeu est alors d'aider les professionnels à cheminer face à l'indétermination dans laquelle ils sont pour agir dans cette situation (Dewey, 1967). Pour cela, identifier les "dimensions agissantes" de la situation et les reconfigurer est une possibilité pour lui permettre d'agir efficacement dans cette situation. Ces dimensions, agissantes définies par (Mayen et al., 2010) comme les " variables essentielles à prendre en compte dans l'activité ».

Les dispositifs construits dans le projet CHANGER s'inspirent de ces éléments et reprennent la proposition de Robert-Guillot (2015) d'animer les échanges entre conseillers en créant des mises en milieu des situations sociales de conseil dans le dispositif d'échange. Pour cette auteure, la mise en milieu est un "moyen de l'action didactique (...) Elle permet i) au professionnel de rendre compte de son travail par la mise en récit d'une situation éprouvée adressée à autrui [...], ii) de connecter la dimension individuelle de l'activité à la dimension collective du travail par la montée en généralité et la mise en patrimoine. Elle fournit un cadre pour reconfigurer l'action » (Robert-Guillot, ibid., p. 102).

\subsection{Le cas d'étude : un dispositif d'échange entre conseiller instauré au niveau régional}

11 Parmi les dispositifs mis en place dans le cadre de CHANGER, nous avons analysé plus particulièrement l'un d'entre eux qui a été conçu et animé à l'échelle d'une région, regroupant des conseillers appartenant à différentes Chambres d'Agriculture au sein de cette région. Onze jours ont été négociés avec les organisations employeuses sur une durée de trois ans pour travailler entre conseillers sur le métier. Deux conceptricesanimatrices (par la suite nommées $\mathrm{C}-\mathrm{A}$ ) ont conçu et animé ce dispositif et ont réparti ces onze jours sur six séminaires tous les six mois, soit environ 2 jours par séminaire. Les C-A ont déjà participé à un dispositif d'échange, au sein du projet de rechercheaction décrit dans Robert-Guillot (2015). Cette expérience, de leur propre aveu, a nourri leurs regards et la conceptualisation qu'elles ont de leur propre métier et de ce qui évolue dans le conseil en agriculture. Elle leur a permis de vivre des modalités d'animation dont elles se sont inspirées pour penser leur propre animation. Enfin, ces C-A ont aussi été parties prenantes de l'élaboration du guide Agroseil (Cerf, Omon, Guillot, Olry, \& Petit, 2013), outil conçu à l'issue de ce projet de recherche-action pour aider les conseillers dans l'analyse et l'identification des dimensions agissantes des situations de travail. Elles sont donc un peu familiarisées à la manipulation de ce guide et à l'analyse de l'activité qui est au cœur de celui-ci.

Outre les C-A, ce dispositif mobilise une dizaine de conseillers ( 3 hommes et 7 femmes) ayant entre 4 et 28 années d'expérience professionnelle. Ils exercent les métiers de conseiller agricole en grandes cultures / conseiller agronomie-environnement / 
conseiller en animation et développement local. Ils ont été invités à participer à ce dispositif sans nécessairement avoir choisi eux-mêmes d'y participer, bien qu'ils perçoivent tous des problématiques dans la façon d'exercer leur métier sans pour autant identifier comment faire évoluer leurs activités.

Ce dispositif s'est construit chemin faisant autour de situations sociales de conseil rencontrées par les participants durant les trois ans et de leurs mises en milieu par le travail d'animation réalisé par les C-A. Ces mises en milieu s'opèrent selon deux modalités (Duhamel, 2019). La première repose sur la mise en récit par les professionnels d'une expérience en acte et en situation comme proposée par RobertGuillot (2015). Ces récits sont produits, analysés et débattus dans le collectif. Ce travail collectif est étayé par un outil commun, l'Agroseil (Cerf et al., ibid.) permettant aux professionnels d'analyser le couplage qui a lieu entre le professionnel et la situation, pour repérer les dimensions agissantes des situations et s'en saisir pour agir différemment. La seconde modalité consiste, pour les C-A, à créer, dans l'espace protégé qu'elles ont construit au sein du dispositif, des perturbations par rapport aux situations sociales usuelles dans le conseil agricole. Ces perturbations sont introduites pour suspendre l'activité usuelle dans ces situations sociales, et permettre aux conseillers de faire l'expérience d'autres couplages possibles en ayant à trouver comment agir dans ces situations perturbées. Les C-A ont particulièrement créé deux perturbations dans le cadre de la situation sociale qu'est le tour de plaine ${ }^{3}$, situation emblématique de l'exercice du métier de conseiller en production végétale (Robert-Guillot, ibid.).

\section{Une observation participante et un suivi longitudinal du dispositif}

14 Au sein de ce dispositif, nous avons mis en place une observation participante ${ }^{4}$ (Olivier de Sardan, 1995) et une méthode de suivi longitudinal pendant les trois années du dispositif, entre deux espaces : celui du dispositif d'échange d'une part (espace 1) celui des expérimentations en situation de travail que tentent les conseillers pour reconfigurer la situation de conseil avec les agriculteurs d'autre part (espace 2). Dans chacun de ces espaces, le recueil de données s'est réalisé sur trois temps autour des situations d'échange, celles créées dans le dispositif au sein de l'espace 1, celles entre conseiller et agriculteurs au sein de l'espace 2 : un temps d'entretien en amont, un temps de suivi, un temps de débriefing. Lors du suivi dans chacun des espaces, des photos et vidéos ou/et des enregistrements audio ont été réalisés. Par ailleurs, le premier auteur a tenu un carnet de bord pendant la durée du dispositif en lien avec sa posture d'observatrice participante.

15 L'analyse est orientée par le souhait de saisir les interactions possibles entre les deux espaces pour appréhender le développement professionnel des conseillers en considérant ce dernier comme un " processus graduel d'acquisition et de transformation des compétences et des composantes identitaires conduisant progressivement les individus et les collectivités à améliorer, enrichir et actualiser leur pratique, à agir avec efficacité et efficience dans les différents rôles et responsabilités professionnelles qui leur incombent, à atteindre un nouveau degré de compréhension de leur travail et s'y sentir à l'aise»" (Uamariya \& Mukamurera, 2005, p. 12). C'est cette dimension graduelle et progressive qui nécessite 
la mise en place d'un suivi longitudinal pour observer le processus « en train de se faire ».

Dans ce qui suit, nous restituons l'analyse que nous faisons d'une partie des données recueillies, plus particulièrement celles recueillies à l'occasion du travail fait dans le dispositif (espace 1) ou dans des expérimentations par les conseillers (espace 2) en nous focalisant dans les deux cas sur la situation sociale du «tour de plaine ». L'analyse de ces données s'est faite en plusieurs temps. Au sein du premier espace, nous avons analysé la conception de deux situations sociales de tour de plaine "perturbées " par les C-A, en précisant les objectifs didactiques poursuivis et la méthodologie mise en place. Puis nous avons identifié la façon dont les échanges autour de ces situations permettent aux conseillers d'analyser l'activité en tour de plaine, de repérer les dimensions agissantes et de s'interroger sur la façon de les organiser pour imaginer ou oser faire autrement avec leurs agriculteurs lors de tours de plaine selon les marges de manœuvre (Coutarel, Caroly, Vézina, \& Daniellou, 2015) qu'ils envisagent, tout en cherchant à capter la façon dont cela est rendu possible par les mises en milieu opérées par les C-A. Pour cela, nous avons cherché à capter la compréhension de la façon dont l'objet de la relation de service peut être travaillé dans les situations entre conseillers et agriculteurs, et la construction par les conseillers, lors des échanges, de situations de travail susceptibles d'étendre la compréhension de cette situation sociale de conseil au profit des agriculteurs et pour eux-mêmes.

$17 \mathrm{Au}$ sein du deuxième espace, notre analyse a porté sur la façon dont les conseillers comprennent et analysent leurs activités en situation, et y repèrent des opportunités pour imaginer et réaliser une autre façon de conduire l'interaction avec les agriculteurs pour aborder avec eux l'agroécosystème. Cette analyse nous permet de capter la réalisation du potentiel de développement des situations créées par les C-A tout en cernant comment la situation expérimentée devient à son tour une situation potentielle de développement pour le conseiller voire pour les agriculteurs. Pour chaque conseiller nous avons : (i) identifié les objectifs et les actions à réaliser que le conseiller énonce dans les entretiens amont; (ii) analysé le déroulé en situation, la manière dont les interactions avec les agriculteurs peuvent reconfigurer la situation et la façon dont le conseiller le prendra en charge ; (iii) relevé, dans les entretiens ex-post, la manière dont le conseiller relate son vécu du point de vue de la façon dont l'activité réalisée questionne l'objet du travail à traiter et la relation qu'il a avec les agriculteurs, tout en discutant la façon dont il analyse la réorientation de l'activité en situation si elle a eu lieu lors d'événements qui perturbent le cours de son action. Nous avons également analysé l'insertion de cette situation dans un processus temporel d'accompagnement des agriculteurs au changement de pratiques : pour cela nous avons analysé les verbatim tant en situation qu'en entretien sur l'inscription de la situation dans une temporalité du travail fait entre le conseiller et les agriculteurs.

Le dispositif tel qu'il a été conçu par les C-A crée des itérations entre ces deux espaces pour permettre aux professionnels de questionner leurs situations et partager leurs réflexions face non seulement aux situations sociales qu'ils vivent dans le dispositif, mais également sur le terrain et entre eux. Ces échanges entre les temps "formatifs » et ce qui est tenté au niveau individuel sont perçus comme permettant de nourrir une réflexion croissante et constante au service d'une prise de recul et de l'analyse de l'activité dans des situations perçues par les conseillers comme étant plus complexes. Les C-A n'imposent pas aux conseillers de tenter quelque chose. Ces derniers peuvent 
ne rien expérimenter ou identifier les situations où ils se sentent autorisés et avoir les marges de manœuvre pour le faire. Cela peut advenir immédiatement ou non après qu'ils aient vécu les mises en milieu proposées par les C-A. Le suivi longitudinal nous permet alors de saisir les liens entre ce qui s'est joué dans les mises en milieu et ce qui s'expérimente. Dans ce qui suit, nous ne rendons pas compte de la façon dont ces itérations entre les deux espaces sont vécues par les conseillers et soutiennent dans la durée leur développement professionnel (voir Duhamel, 2019). Nous restituons plus spécifiquement la façon dont s'opère l'itération entre les situations de tours de plaine "perturbées » proposées par les C-A et ce qu'expérimentent certains conseillers avec leurs agriculteurs en se saisissant de ces mises en milieu créées par les C-A.

\section{Une capacité différenciée à s'approprier le potentiel de développement de situations sociales nouvelles}

Les conceptrices-animatrices sont conscientes de l'importance actuelle des références techniques et de la technique agronomique dans les situations sociales de conseil usuelles et de la difficulté à construire un rapport renouvelé aux savoirs agronomiques. Pour cela, en accord avec l'orientation du projet CHANGER, elles font le choix de proposer un espace protégé dans lequel le métier dans sa globalité sera abordé travaillant ainsi la triple extension des situations de conseil. Dans ce cadre, les C-A ont opéré des mises en milieu des situations sociales de conseil que sont le tour de plaine, pour, disent-elles, "décoiffer, décaler et déplacer» les conseillers par rapport à leurs routines de conseil. Pour rendre compte de la façon dont ces mises en milieu rendent ces situations apprenantes et crée les conditions permettant aux conseillers de s'approprier le potentiel de développement de ces situations, nous présentons l'analyse de ce qui se joue dans le collectif lors de ces mises en milieu puis montrons comment ce qui s'est joué là est saisi (on non) par les participants pour développer de nouvelles façons d'exercer et de penser l'agir efficace en situation sociale de conseil.

\subsection{Le collectif : un espace d'appropriation de l'idée de mise en milieu d'une situation « perturbée » et " perturbante » ?}

20 L'ayant expérimenté elles-mêmes dans un dispositif de formation-action (voir RobertGuillot, 2015), les deux C-A perçoivent l'intérêt d'un regard « décalé » sur une situation habituelle de travail pour permettre aux conseillers de se questionner sur leurs propres routines et envisager d'exercer " différemment » leur activité. Elles souhaitent que les conseillers soient capables, grâce à leur participation au dispositif qu'elles mettent en place, d'identifier ce qui évolue et ce qui "pose question" dans leurs situations de travail pour identifier « ce sur quoi » ils peuvent agir dans leur action de conseil avec les agriculteurs.

\subsubsection{Les mises en milieu opérées : manipulation des dimensions des situations de tour de plaine}

21 Dans cette perspective les C-A prennent comme situation le tour de plaine qui est, comme l'ont pointé Guillot et al. (2013) une situation emblématique pour les conseillers travaillant dans le domaine des productions végétales. Les C-A imaginent deux mises en 
situation dans lesquelles les conseillers seraient « décalés, voire perturbés» [au sens d'un conflit sociocognitif] par rapport à une situation habituelle pour susciter une posture réflexive et de prise de recul quant à leurs propres routines. Comme le met en évidence le tableau 1 ci-dessous, les $\mathrm{C}-\mathrm{A}$ créent une première mise en milieu qui met les conseillers dans un environnement dans lequel ils sont "empêchés": face à une culture de cassis qu'ils ne connaissent pas, étant spécialisés sur les grandes cultures (blé, orge, colza, maïs...), et en l'absence de l'agriculteur qui habituellement leur donne les informations sur ce qu'il a fait dans la parcelle, ils ne peuvent mobiliser leurs raisonnements techniques habituels. L'enjeu ici est de les amener à questionner la situation différemment sur le plan agronomique et de mettre en débat la façon dont les conseillers font un diagnostic agronomique sur une parcelle. Puis, dans un second temps, au regard de ce que les conseillers renvoient lors des séminaires, les C-A proposent une seconde mise en milieu afin de jouer sur les objectifs de leur animation et d'amener les conseillers à travailler un autre objet, leur façon de questionner les agriculteurs. Il est donc nécessaire que l'agriculteur soit présent. L'enjeu est de faire évoluer le questionnement agronomique pour faire expliciter à l'agriculteur la façon dont il pense et conduit son système de culture ${ }^{5}$, pour mettre à jour ses règles de décisions et objectifs. Cela est différent du questionnement classique en tour de plaine où l'agriculteur est interrogé sur ce qu'il a fait jusqu'ici dans sa parcelle pour comprendre l'état de la parcelle et lui suggérer les actions à mettre en place tout en lui rappelant les règles de décisions techniques issues des références. L'enjeu du nouveau questionnement et de favoriser une dynamique d'échange permettant in fine, de faire émerger la conceptualisation qu'à l'agriculteur de son système et de sa conduite. Pour éviter que les conseillers soient « tentés » de retomber dans la configuration habituelle des conseillers qui travaillent avec des agriculteurs dits " conventionnels", les C-A ont choisi un agriculteur qui exerce son activité en agriculture biologique. L'impossibilité de recourir à des intrants chimiques oblige alors une vision plus systémique pour analyser une problématique et la mettre en regard de ce que recherche l'agriculteur comme résultat. Enfin, les C-A organisent cette seconde mise en milieu en attribuant des rôles différents aux conseillers répartis alors en trois groupes: les "questionneurs » devaient interroger l'agriculteur concernant son système de culture et ses règles de décision en mobilisant "un questionnement agronomique »; les « rapporteurs » devaient de prendre des notes sur les connaissances recueillies pour retracer le système de culture de l'agriculteur; les "observateurs" devaient se "mettre à distance » et analyser les échanges qui avaient lieu entre les questionneurs et l'agriculteur et ce qu'ils ont ou pas permis d'atteindre comme raisonnement agronomique (voir Tableau 1). 
Tableau 1 : deux tours de plaine " décalés » construits par les C-A : objectifs et modes opératoires. Table 1: two "unconventional" tours of the field developed by the designers-facilitators: objectives and operative modalities

\begin{tabular}{|c|c|c|}
\hline & Situation 1-octobre 2014 & Situation 2 - novembre 2015 \\
\hline $\begin{array}{l}\text { Conditions de la } \\
\text { mise en milieu }\end{array}$ & $\begin{array}{l}\text { Culture de cassis } \\
\text { Automne } \\
\text { Absence de l'agriculteur }\end{array}$ & $\begin{array}{l}\text { Culture végétale en agriculture biologique } \\
\text { Automne } \\
\text { Présence de l'agriculteur }\end{array}$ \\
\hline Objet travaillé & Diagnostic agronomique & Questionnement agronomique de l'agriculteur \\
\hline $\begin{array}{l}\text { Objectifs visés } \\
\text { dans la situation }\end{array}$ & $\begin{array}{l}\text { Etre en réflexion sur les routines de travail en tour de } \\
\text { plaine dans ce que les conseillers cherchent à travailler } \\
\text { avec les agriculteurs et les informations qu'ils recueillent } \\
\text { sur le terrain pour se représenter la situation agronomique } \\
\text { conduisant à un diagnostic agronomique. }\end{array}$ & $\begin{array}{l}\text { Etre en réflexion sur le questionnement qui peut } \\
\text { être fait auprès de l'agriculteur pour comprendre son } \\
\text { raisonnement agronomique sur son système de } \\
\text { culture et ses règles de décisions. }\end{array}$ \\
\hline $\begin{array}{l}\text { Objectif sous- } \\
\text { tendu }\end{array}$ & $\begin{array}{l}\text { Revisiter, questionner ses pratiques de conseil, ses routines } \\
\text { Aller vers un "conseil à froid " } \\
\text { S'appuyer sur une réflexion plus globale de ce qui se joue } \\
\text { identifier les différentes marges de manœuvre mobilisable } \\
\text { l'identification de différents leviers agronomiques " pour m } \\
\text { l'environnement. }\end{array}$ & $\begin{array}{l}\text { e dans une situation avec ou sans l'agriculteur, pour } \\
\text { pour permettre de travailler avec les agriculteurs à " } \\
\text { mettre en place des pratiques plus respectueuses de }\end{array}$ \\
\hline Mode opératoire & $\begin{array}{l}\text { Les conseillers étaient " libres " d'aller dans la parcelle Les } \\
\text { pour observer et recueillir des informations sur la obse } \\
\text { situation agronomique pour en établir un diagnostic recu } \\
\text { agronomique. L'ensemble des informations recueillies l'agr } \\
\text { était discutées ensemble dans le collectif (séminaire infor } \\
\text { suivant) }\end{array}$ & $\begin{array}{l}\text { conseillers étaient répartis en } 3 \text { rôles : questionneur, } \\
\text { servateur, rapporteurs. Les } 3 \text { questionneurs devaient } \\
\text { ueillir des informations sur le système de culture de } \\
\text { riculteur et ses règles de décision. L'ensemble des } \\
\text { ormations recueillies était partagé le lendemain }\end{array}$ \\
\hline
\end{tabular}

\subsubsection{Ce qu'apprennent ou retiennent les conseillers de ces mises en situation perturbées}

Lors de la première mise en milieu, les conseillers évoquent immédiatement que la situation est " perturbante », car ils notent l'absence de l'agriculteur, le fait d'être dans une culture différente (cassis) et que cela se fasse à l'automne, époque à laquelle ils ne font pas habituellement de tour de plaine. Ces conditions ont, dans un premier temps, amené les conseillers à ne pas savoir quoi observer ni comment. Puis en s'appuyant sur une observation micro et macro de la parcelle et de son environnement, ils ont progressivement recueilli des informations pour établir un diagnostic agronomique (présence d'autres plantes entre les pieds de cassis, présence des insectes et identification de leur fonctionnalité, présence d'arbres aux alentours et de leurs impacts probables sur la parcelle, etc.). En manipulant les dimensions des situations, les C-A ont perturbé l'exercice habituel du tour de plaine pour les conseillers. 
Figure 1 : conseiller à la recherche d'indices pour porter un diagnostic dans la situation perturbée (champ de cassis).

Figure 1: advisor looking for relevant information to make a diagnosis in the "unconventionnal" situation (blackcurrant field)

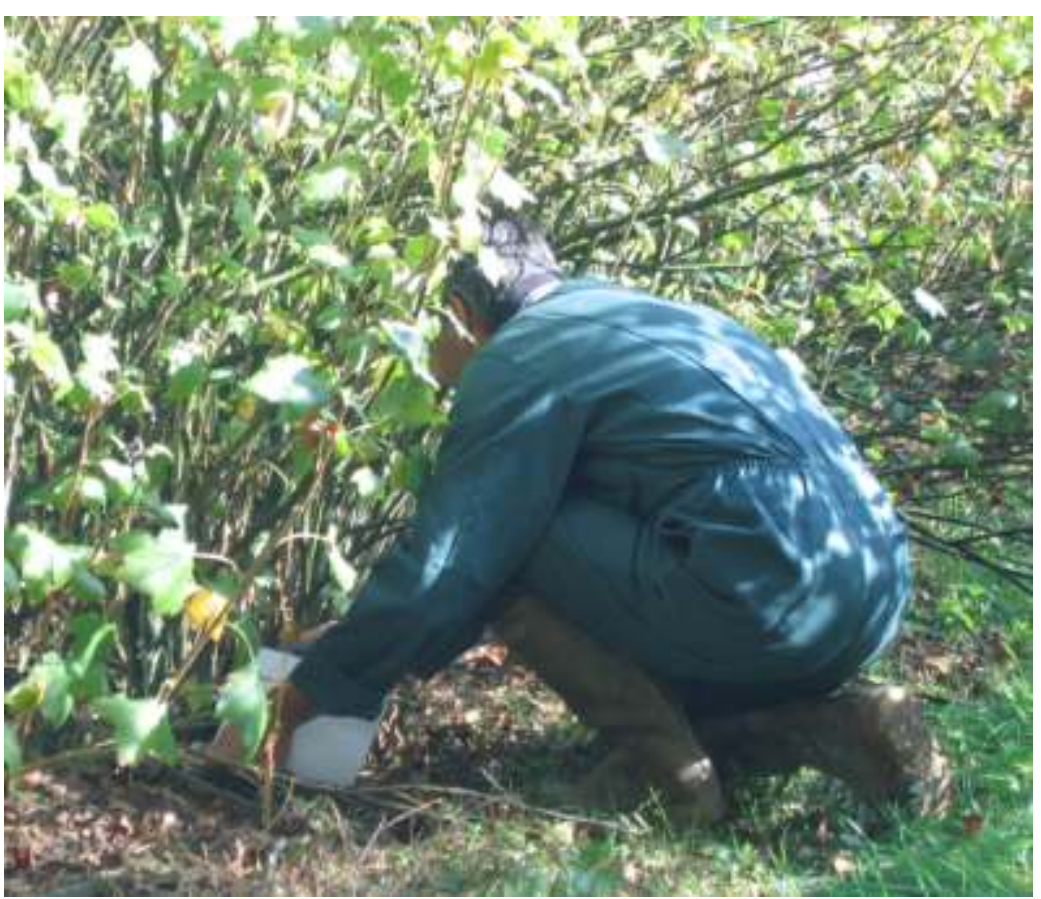

Photo réalisée par P. Olry

Cela amène alors les conseillers à chercher des informations sur lesquelles ils ont prise pour analyser la parcelle. Ce faisant, ils s'ouvrent à l'observation micro et macro de la parcelle pour prélever des indices agronomiques et se questionnent sur la façon dont ces indices observés en situation peuvent agir les uns sur les autres pour définir des problématiques et construire un diagnostic agronomique. Lors du débriefing, cette situation vécue comme " perturbante » est aussi vue comme permettant également « de se poser toutes les questions [agronomiques], car on n'avait pas de problématique ». Comme l'explique un conseiller «dans une situation qu'on ne connait pas du tout " cela permet de faire plus d'observations et « d'identifier des choses. Cela oblige à observer et on voit alors pas mal de choses".

La perturbation ouvre la possibilité d'une situation apprenante sur deux plans. Les conseillers soulignent que "l'absence de l'agriculteur nous a poussés à discuter, le dialogue n'a pas été bridé par les informations amenées par l'agriculteur » et qu'ils ne "se seraient pas posés autant de questions s'il avait été là ». Ils réalisent ainsi qu'ils peuvent modifier la façon dont ils construisent le diagnostic agronomique : plutôt que de le faire à partir des pratiques réalisées par l'agriculteur et que ce dernier leur énonce, ils peuvent le faire en portant leur regard sur des indices révélateurs de l'histoire culturale de la parcelle. Par ailleurs, l'échange qu'ils ont eu sur les informations qu'ils ont recueillies leur fait prendre conscience qu'ils peuvent sans doute interagir autrement avec les agriculteurs, adopter une autre posture. Un conseiller dira que «mettre les gens en situation d'être conseillers, permet qu'ils se lâchent et arrivent à des propositions plus complètes ». La posture est une question importante chez un autre conseiller pour qui cette situation a permis d'effacer «la notion d'expertise et d'expérience, [que] ce sont les réflexes de recherche qui ont 
permis de voir. Que même sur quelque chose qu'on ne connait pas, chacun peut puiser la méthode pour s'en sortir ». Enfin, une conseillère dira que cette "technique pour animer permet de délier les langues [le fait] de placer les gens comme acteurs ».

Lors de la seconde situation, le questionnement préparé en collectif avant de se rendre sur le terrain pousse les trois questionneurs à poser des questions sur le système d'exploitation de l'agriculteur et sur son système de culture. Néanmoins, dans le cours du questionnement ils ont peu cherché à identifier les règles de décision de l'agriculteur et sont restés dans leurs routines habituelles d'exercice visant à identifier ce qui a été mis en place plus que ce qui oriente ce qui est mis en place. À titre d'exemple, une questionneuse (Q2) est intervenue dans l'échange pour exprimer une crainte qu'elle avait quant au choix variétal arrêté par l'agriculteur. Elle dit qu' « elle n'aurait pas fait ce choix variétal» compte tenu des risques liés à cette variété dans un contexte d'agriculture biologique. À ce moment-là un autre questionneur essayera de rediriger l'échange sur les règles de décision que prend l'agriculteur dans le pilotage de ses cultures.

Dans le débriefing de la situation, Q2 remet en cause le choix variétal de l'agriculteur [qu'il a fait seul], et met en avant le fait qu'elle va peu chez les agriculteurs « quand tout va bien » et que si elle se rend chez l'agriculteur elle doit « lui apporter quelque chose ». Le collectif, lui, met en avant que le "questionnement de Q2 a été très technique, en décalage par rapport à ce que les cassis nous ont appris ». Le débat tourne alors autour de la posture que peut/doit avoir le conseiller : oscillant entre apports scientifiques et techniques et co-construction du conseil. Les conseillers identifient que la posture habituelle n'est ici pas adéquate au regard de la demande des C-A. Ils font le parallèle avec leurs situations de conseil dans lesquelles ils " ne sont plus là que pour du conseil technique descendant ». Ce débriefing a également fait émerger la question de "ce qu'il est attendu d'un conseiller " en particulier le fait que les agriculteurs attendent «de la technique». La situation permet ainsi aux conseillers de s'interroger sur les limites d'une relation fondée sur le transfert d'expertise, tout en n'étant pas tous d'accord sur la façon de les dépasser dans leurs propres situations professionnelles.

Appuyés par la dynamique collective, les conseillers se sont exprimés sur ces situations et évoquent ce qu'ils pourraient faire de ces situations dans leur propre activité. Ainsi, plusieurs conseillers notent que ce type d'animation, de questionnement, de situations peut être intéressant à travailler pour aider les agriculteurs à se détacher d'une vision immédiate et à court terme de la gestion d'une parcelle. Pour d'autres, en revanche, la rupture avec leurs situations habituelles est trop forte et cela ne permet pas d'envisager une application. En cela, chacun prend en charge les mises en milieu créées par les C-A et en fait quelque chose pour soi, mais cela peut prendre différentes formes selon les contextes situationnels et les conseillers.

\subsection{L'individuel : un « espace » d'expression pour réaliser le potentiel de développement des situations pour autrui / pour soi}

Ayant vécu ce qui se joue pour eux dans le cadre des deux mises en milieu créés par les C-A, les conseillers agricoles sont " plongés » dans leur propre univers de travail et se saisissent chacun à leur façon de ces mises en milieu au regard de leur propre contexte d'exercice. Dans ce qui suit, nous mettons en lumière la façon dont certains conseillers se sont approprié des situations apprenantes vécues en collectif pour mettre en place 
de nouvelles façons d'exercer leur activité. De notre analyse, nous dégageons, dans la diversité observée parmi les conseillers, trois manières d'engager une reconfiguration de leur situation d'échange avec les agriculteurs. Notons cependant que, dans le collectif que nous avons suivi, deux conseillers n'ont pas testé ou mis en place de nouvelles façons de faire même s'ils disent avoir pris conscience de dimensions agissantes à l'occasion du travail réalisé dans le dispositif, mais, disent-ils "ce n'est pas ce qu'attendent mes agriculteurs", "ils attendent de moi des réponses techniques et immédiates ».

\subsubsection{Des conseillers qui opèrent une « transposition méthodologique »} une situation s'inspirant de ce qu'elles ont vécu lors de la première mise en milieu. Le BAC est un périmètre dans lequel la qualité de l'eau est observée avec attention, car polluée en partie par l'activité agricole. Pour y remédier, les agriculteurs doivent développer des pratiques plus respectueuses de l'environnement dans un contexte de travail qui s'organise entre eux, l'agence de l'eau, la collectivité qui utilise l'eau du captage, et, dans le cas présent, la Chambre d'agriculture qui assure l'accompagnement des agriculteurs et la mise en œuvre du plan d'action validé par l'agence de l'eau et destiné à améliorer la qualité de l'eau au captage.

La première conseillère souhaite réinvestir le temps de réflexion et de prise de recul qu'elle a vécu dans le collectif, car ce fut l'occasion « de se poser des questions que l'on ne se serait jamais posées avant ». Elle souhaite créer cette ouverture pour que les agriculteurs puissent s'ouvrir à des réflexions qui inscrivent les parcelles sur leur territoire, en l'occurrence dans le BAC, pour penser cette double gestion de la parcelle dans le temps de la campagne et dans le temps longitudinal de la protection de l'eau en diminuant l'utilisation des produits phytosanitaires. Pour cela, elle souhaite valoriser les connaissances et les expériences des agriculteurs au niveau des parcelles et du territoire, signe d'une gestion spatio-temporelle. Comme les C-A l'ont fait pour les conseillers, elle part du postulat que rendre les agriculteurs acteurs dans la compréhension des problématiques de la situation peut les amener plus facilement à être acteurs de leurs changements de pratiques. Cette conseillère construit en deux temps sa mise en situation :

- Un premier temps où les agriculteurs sont invités à réfléchir en sous-groupes en salle à partir de cartes IGN, sur l'identification des ruissellements dans les parcelles, les actions déjà mises en place et ce qu'il serait possible de mettre en place.

- Dans un second temps elle souhaite s'appuyer sur les propositions des agriculteurs les plus facilement appropriables et réalisables. Elle les conduit sur le terrain pour aller voir des parcelles dans lesquelles des actions sont déjà mises en place ou à construire à partir de celles identifiées par l'exercice précédent. L'objectif est de rendre opérationnelles certaines pistes d'action proposées en collectif.

Sur le terrain, les agriculteurs refusent les propositions vues en salle orientant ainsi la situation vers une discussion fermée. Sentant qu'elle ne parviendra pas à construire des pistes de solutions avec eux, elle réoriente son activité en écoutant les problématiques des agriculteurs entre «leur métier qui devient de plus en plus dur et la problématique du BAC qui s'ajoute» (les agriculteurs mentionnent que les deux orientations sont difficilement compatibles). 

leurs réflexions habituelles pour imaginer autrement leur parcelle en entrant par le territoire et la problématique du ruissellement de l'eau. Le travail réalisé en amont de la visite de terrain lui a permis d'identifier les marges de manœuvre envisagées par les agriculteurs à qui elle a ensuite proposé de les mettre en œuvre. Elle a posé les conditions d'une situation apprenante en deux temps pour les agriculteurs en conjuguant capacité de projection et mise en œuvre opérationnelle. Elle a ainsi identifié un certain potentiel de développement de cette situation mais ne parvient pas, sur le terrain à ce que les agriculteurs passent à l'action. d'agriculteurs et de conseiller impliquent pour elle. Tout en souhaitant reprendre «cette méthode d'animation [vue dans le dispositif]» pour une situation sur un BAC nouvelle pour elle, cette conseillère exprime la difficulté qu'elle ressent :

"J'ai été confrontée à un mode de conseil totalement différent, et c'est un peu perturbant d'ailleurs. Parce que comment dire, quand on va en groupe technique, les agriculteurs on les connait, on connait leur cas, ils ont des questions, souvent précises et pour un but qui est à peu près le même pour tous c'est d'avoir le meilleur résultat, la meilleure marge à la fin ». Dit autrement, dans le BAC, le collectif d'agriculteurs n'en est pas un, ils sont réunis, car leurs parcelles sont dans la zone $d u B A C$, ils ne sont pas réunis par une orientation technique partagée, et la conseillère ne les connaît pas. Elle partage le fait que lorsqu'elle agit sur le $B A C$, elle "ne sait pas trop ce qu'on fait là et pourquoi on est là, et qu'est-ce qu'ils [les agriculteurs] attendent et si ce qu'on fait va amener à quelque chose ».

Elle se questionne sur le sens de son activité: «sommes-nous un bon conseiller si on n'apporte pas de concret immédiat?». Cette conseillère met en avant, qu'elle se "sent capable» d'envisager ce type de situation décalée auprès de collectifs d'agriculteurs organisés autour des BAC, car «ce sont des groupes contraints » et la problématique de la qualité de l'eau s'y prête. En revanche, pour ses groupes d'agriculteurs habituels « plus techniques » elle ne «se sent pas capable» d'aborder son activité sous ce nouvel angle. Dans ce cadre-là, les agriculteurs l'attendent pour quelque chose de précis et ne sont pas en demande "de travailler autrement ou de penser différemment leur approche de leur système ». Cette conseillère va construire une situation projetant à long terme les agriculteurs du BAC («quels sont selon vous les défis de la ou les prochaines campagnes pour les réussites de vos cultures?»). Dans un second temps, elle leur propose de faire le parallèle avec ce qu'ils font actuellement sur leur parcelle. L'objectif sera de construire «un nouvel itinéraire technique ${ }^{6}$, avec les agriculteurs, appuyé sur des leviers agronomiques.

Ces deux conseillères ont mis en place deux situations dont l'animation est directement issue des mises en milieu vécu en collectif. Elles ont transposé l'objectif de rendre les professionnels acteurs de la situation pour les amener à avoir une réflexion sur leurs parcelles à la fois dans un espace temporel et spatial plus large, au regard de leurs pratiques actuelles. Pour cela, elles ont mis en évidence différentes dimensions agissantes de la situation à construire et se sont appuyées dessus pour en faire un levier de réflexion. En ce sens, elles ont analysé et manipulé les dimensions agissantes de la situation en "transposant " un outil d'animation dont elles ont perçu, pour ellesmêmes, les effets. Il est intéressant de noter que pour la seconde conseillère, ce processus n'est possible que dans des situations comme le BAC et qu'elle estime ne pas avoir le mandat de le faire dans ses groupes techniques habituels. 


\subsubsection{Un conseiller qui s'approprie et réalise un jeu sur l'espace-temps de la mise en milieu}

Un conseiller s'est saisi de la temporalité du dispositif pour expérimenter plusieurs situations sur le terrain toujours dans le cadre d'un BAC. Chacune des situations ainsi mises en place a été réalisée à la suite des situations vécues en collectif. Ce conseiller doit accompagner techniquement les évolutions de pratiques des agriculteurs pour réduire l'usage de produits azotés ou phytosanitaires grâce à la mise en place de leviers agronomiques. Les agriculteurs de ce territoire sont en attente "d'autre chose que de la technique pure ». Le conseiller l'exprime également en disant que "la chimie arrive au bout [de ce qu'elle peut faire] » et qu'il faut accompagner les agriculteurs différemment pour les engager dans ce processus d'évolution de pratiques ce qui le conduit à «vouloir faire autrement ». Suite à la première mise en milieu du tour de plaine dans les cassis, il crée une première situation en mai 2015 , hors période de surveillance des maladies et insectes. Avec une collègue animatrice de ce BAC, il analyse les dimensions agissantes de la situation et identifie l'objet à traiter: la problématique des adventices ${ }^{7}$. Cette dernière est souvent formulée par les agriculteurs sur le court terme à travers le choix du produit, de la dose et de la date pour traiter. La question des adventices est un enjeu sur le BAC car les produits herbicides sont à l'origine des principales pollutions des nappes phréatiques par infiltration sur ce BAC. Pour ces conseillers, il s'agit de traiter la question sur le long terme, à l'échelle du système de culture, pour ouvrir des possibilités nouvelles quant à la façon de gérer les adventices. Ainsi, ils construisent une situation pour amener les agriculteurs à réfléchir collectivement sur ce qui peut être fait sur une parcelle pour gérer les adventices à court et long terme tout en limitant l'utilisation des produits phytosanitaires. Pour cela, ils associent des temps d'observation et de réflexion, en invitant à réfléchir à court terme (année en cours) et à moyen/long terme sur 3 à 5 ans.

Par cette mise en réflexion collective, le conseiller souhaite que les agriculteurs puissent construire un diagnostic agronomique sur la parcelle et le mettre en relation avec sa gestion. Pour cela les agriculteurs ont pour consigne dans un premier temps d'aller observer une parcelle et d'évaluer l'importance du «salissement» (e.g. une évaluation subjective du nombre et des types d'adventices présentes) d'une parcelle et de son caractère problématique. Il leur est également demandé d'identifier des pistes d'action pour répondre à cette problématique d'adventices tout en ayant en tête la protection de l'eau. Le deuxième temps s'organise autour d'un partage collectif sur le salissement de la parcelle confrontant les agriculteurs à leur propre perception " d'un champ propre » et de l'impact que cela peut avoir sur la qualité et le rendement des cultures. Ils prennent alors conscience qu'avoir un champ "moins propre » à court terme peut avoir un intérêt sur la qualité de l'eau sans pour autant perturber le rendement. Ce faisant, ils réévaluent leurs critères pour apprécier l'impact du nombre et du type d'adventices sur la culture comme sur le système de culture. La question des pratiques agricoles à mettre en œuvre pour répondre à cette problématique, en ayant la double focale de la qualité de l'eau et de la projection des effets à moyen et long terme d'une présence d'adventices (augmentation du stock de graines par exemple) à la parcelle, se révèle néanmoins plus compliquée à aborder. Si le conseiller souhaite que les agriculteurs mobilisent différents leviers agronomiques pour répondre à cette problématique, ces derniers proposent essentiellement des solutions chimiques à court terme (dans les 6 mois futurs). Face aux propositions des agriculteurs d'utiliser tel 
produit, à telle dose et telle date, le conseiller essaie de les amener à se projeter : « oui mais si tu devais penser à 3-5ans ta solution, elle tiendrait?». Les agriculteurs ont retourné la question au conseiller, qui se retrouve alors dans une position de «sachant » dans laquelle les agriculteurs sont en attente "de ce qu'il ferait». Face à une difficulté des agriculteurs à se détacher de la chimie et à se projeter, le conseiller dira en entretien post-situation "qu'ils [lui-même et la conseillère] auraient pu aller plus loin dans le questionnement " même si cette première situation a permis d'approcher une nouvelle façon de questionner l'évolution d'un champ (en termes de salissement et de choix à faire durant l'année à venir).

Une année après, par une construction en trinôme, ce même conseiller propose une situation dans la continuité de la précédente, dans laquelle il souhaite mettre les agriculteurs en position d'acteurs. Cette situation a de nouveau lieu au printemps où il n'est plus possible d'intervenir chimiquement et en l'absence de l'agriculteur qui cultive le champ. Il souhaite faire émerger les raisonnements agronomiques des agriculteurs qui sous-tendent leurs règles de décisions. Pour cela, il organise la situation en trois temps :

i. un premier temps au cours duquel le conseiller rappellera «ce qui fait sens» dans sa présence sur un BAC et l'enjeu autour de la protection de la qualité de l'eau ;

ii. un temps dans les parcelles ou deux groupes d'agriculteurs doivent faire un diagnostic de parcelle et proposer des pistes d'action pour sa gestion future;

iii. un temps d'échanges et de mise en commun des deux groupes.

En situation, dans la première partie, l'intervention inopinée d'un agriculteur contre le travail réalisé sur le BAC qui vise à inciter une réduction de l'usage des pesticides est saisie par le conseiller comme une opportunité pour rappeler le principe qui réunit agriculteurs et conseillers de Chambre pour travailler sur la qualité de l'eau avant que les réglementations les obligent à modifier leurs pratiques. Il saisit cette intervention pour rappeler le sens de ce travail collectif et son souhait que «les évolutions de pratiques viennent d'eux et qu'elles soient cohérentes avec leurs systèmes de culture ». Dans la seconde partie, en sous-groupes, le conseiller accompagne l'un des sous-groupes et conduit les agriculteurs à observer non seulement les adventices, mais également les invitent à resituer cette parcelle dans son environnement spatial ou encore reprend des interrogations des agriculteurs sur la possible résistance du Ray Grass ${ }^{8}$ aux herbicides. En mobilisant un questionnement agronomique, il amène les agriculteurs à dépasser une première analyse "à chaud » et à être en réflexion sur les origines possibles de la problématique, faisant ainsi émerger le raisonnement agronomique des agriculteurs.

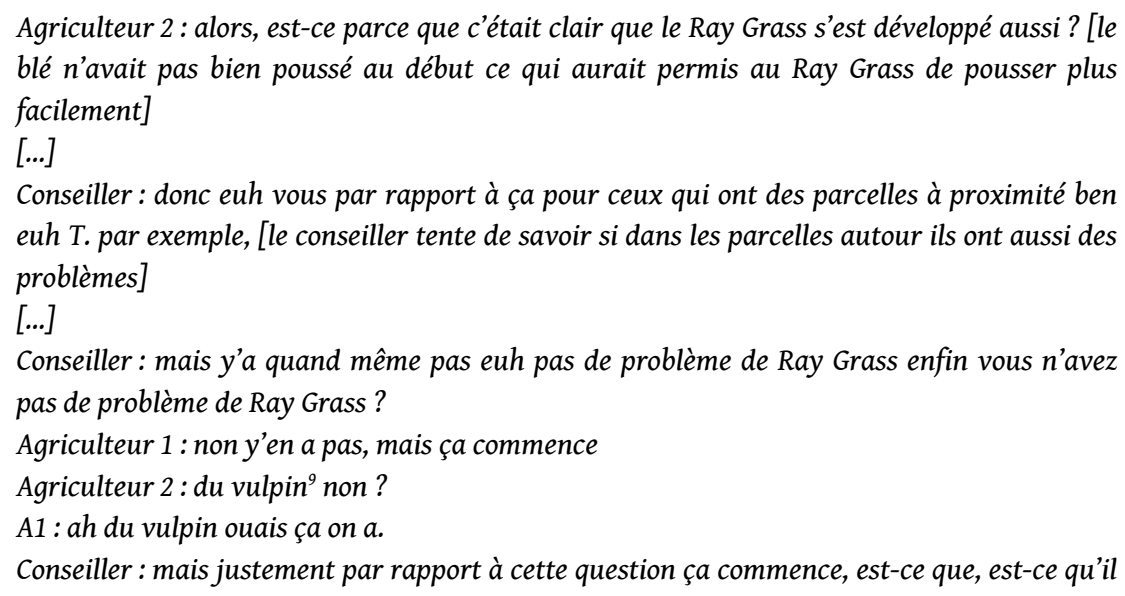


n'y aurait pas, est-ce qu'il y aurait des choses particulières que vous feriez, enfin qu'est-ce que, enfin comment vous géreriez cette parcelle quoi, en sachant qu'il commence à y en avoir du Ray Grass ? Donc est-ce qu'il y a des précautions particulières ou euh?

Agriculteur 3 : on les arrache les unes après les autres [rire]

Conseiller : (note et dit) ok, on les arrache à la main, on a commencé là

Agriculteur 2: des fois c'est dans le programme phyto désherbage c'est en tenir compte c'est tout, t'as pas le choix maintenant, t'as plus le choix parce que ça si tu te laisses envahir dans 5 ans, voilà c'est [je n'entends pas mais s'approche de "tu en as de partout dans le champ »] Conseiller : ouais mais alors toi tu me dis que tu suspectes que c'est des résistances, toi tu me dis c'est peut-être résistant alors est-ce que le phyto euh... est approprié?

Agriculteur 3 : ah ben là on est dans la merde

Conseiller : ah ben ouais mais

A1: ouais mais le Ray Grass c'est spécial hein

A2 : c'est tenace hein, et le problème du Ray Grass c'est beaucoup plus onéreux à traiter pour désherber du Ray Grass que du vulpin et c'est beaucoup plus difficile à détruire. collectif pour engager des agriculteurs non volontaires dans une évolution de pratiques. Dans les deux cas, le conseiller a analysé en amont les situations pour en extraire les buts que lui et ses collègues souhaitaient atteindre et a repéré les différentes dimensions sur lesquelles s'appuyer pour construire une autre approche de son activité. Dans la première situation, par le changement qu'il opère, les agriculteurs ont pris conscience des différences d'appréciation suivant les contextes et résultats visés de chaque agriculteur. La seconde situation rend possibles des échanges autour des raisonnements agronomiques que mobilisent les agriculteurs et des débats entre eux pour analyser une situation et proposer une piste d'action. Le conseiller a organisé autrement la situation pour rendre les agriculteurs encore plus acteurs dans l'observation et l'analyse d'une situation: il propose un travail en sous-groupe et pousse le questionnement agronomique dans les sous-groupes pour faire émerger le raisonnement agronomique des agriculteurs au-delà des "premières analyses ». La préparation qu'il a faite lui permet aussi, face à une dimension émergente en situation (l'intervention négative d'un agriculteur à propos de ce qu'ils font ensemble) d'en faire un levier pour (re)partager le principe commun qui les réunit: l'amélioration de la qualité de l'eau.

41

Ce faisant, le conseiller va au-delà de la transposition d'une technique d'animation comme cela était le cas pour les deux conseillères précédentes. Il s'interroge sur les dimensions des situations, les agence en construisant des conditions de développement et manipule ces dimensions tout en s'appuyant sur les événements émergeant pour réorienter son action. En cela, il s'attache à réaliser des mises en milieu qu'il réitère dans le temps tout en les faisant évoluer pour rendre ces situations d'échange au sein d'un BAC apprenantes pour les agriculteurs. 


\subsubsection{Des conseillers qui produisent des mises en milieu dans des situations collectives nouvelles}

Se saisissant autrement de ce qu'ils ont vécu en collectif, trois conseillers expérimentent différemment des situations. Une conseillère prépare une mise en milieu en analysant les dimensions agissantes et les problématiques auxquelles elle fait face dans son travail avec les agriculteurs sur un BAC (voir Figure 2). Il lui est important de "décaler" les agriculteurs pour "qu'ils puissent penser autrement leurs pratiques agricoles» d'autant plus qu'ils «ne suivent pas vraiment les recommandations du BAC». La conseillère commence par rappeler le principe commun de ce collectif de travail entre la Chambre, l'agence de l'eau et les agriculteurs. Elle propose par la suite de laisser les agriculteurs observer la parcelle en répondant aux questions suivantes :

"Trouvez-vous la maitrise des adventices satisfaisante de votre point de vue dans cette parcelle" ? ; la deuxième question c'est "quels indices vous font dire ça»? La troisième question ben "comment vous les avez observés? " et la quatrième question «que feriez-vous dans cette parcelle si vous la repreniez en termes de rotation, travail du sol, tout ce qui est stratégique au niveau de l'itinéraire technique »?

L'agencement des questions a été construit pour permettre de faire émerger un fil de réflexion sur ce qui est observé dans la parcelle et la mise en avant d'un raisonnement agronomique pour établir un diagnostic agronomique. Lors des interventions des agriculteurs, la conseillère va les questionner davantage pour les amener à approfondir leurs réponses pour expliciter pourquoi ils en viennent à dire ceci, faisant émerger leurs raisonnements agronomiques. Elle a conçu des supports pour permettre aux agriculteurs de noter et discuter.

Oui on a essayé d'aller au fond de la réflexion.

C'est difficile, ce sera difficile, car il faut revenir à des bases agronomiques et leviers qu'ils faisaient il y a des années parce qu'il n'y avait pas tant que produits (entretien postsituation)

Figure 2 : représentation de l'enchainement de la situation de conseil, et des supports intermédiaires mobilisés, conçue par $\mathrm{C}$ autour de la question de la gestion des adventices et la pollution de l'eau par les herbicides dans le Bassin d'Alimentation de captage.

Figure 2: the advisory situation developed by the designers to deal with weed management and water pollution using herbicides in a water catchment area

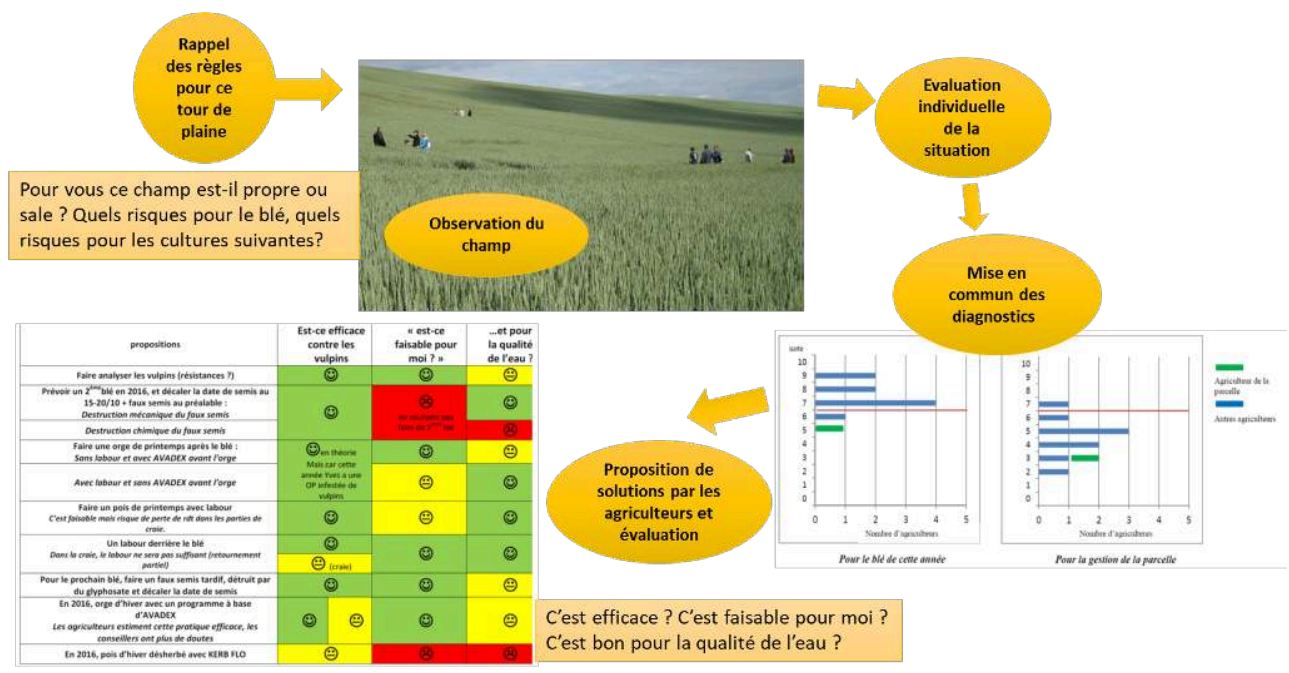

La conseillère va aussi mobiliser les expériences des agriculteurs pour qu'ils puissent partager leurs connaissances. Elle s'appuie particulièrement sur un agriculteur qui a 
une très forte tolérance aux adventices dans ses parcelles et qui mobilise différents leviers agronomiques pour les gérer, car pour lui "les dommages que les adventices pourraient causer sont compensés par le gain qu'il a à effectuer moins de traitements ». À l'inverse, un autre agriculteur, traitant énormément ces parcelles, intervient pour demander «ce que préconise la Chambre». La conseillère s'appuie alors sur la réflexion collective des agriculteurs plutôt que d'apporter une réponse technique. À la fin de l'exercice, ce même agriculteur viendra dire que ce type d'exercice pourrait être réalisé dans une de ses parcelles, car il est confronté à une résistance aux produits chimiques de ses adventices qu'il «ne sait plus comment gérer ». Cela constitue un indice de la façon dont l'agriculteur se saisit du potentiel de développement de la situation ainsi créée par la conseillère.

Au-delà de cette situation, cette conseillère précise que c'est l'ensemble de sa propre façon de travailler qui est revue :

Et puis ben le changement c'est maintenant donc là on est toujours dans un champ, sauf que là c'est les agriculteurs qui vont eux-mêmes aller voir leurs champs, faire leur diagnostic, moi je suis à côté, je bois un coup et ensuite on fait le bilan c'est moi plus qui vais questionner ce qu'ils ont vu, les faire échanger et faire plus de retours que ce que moi je vais leur en rapporter. Même si bien sûr il reste toujours une partie où moi j'interviens techniquement mais après.

Donc ça, ça m'a permis de me rassurer sur ce que je faisais sur ma façon de faire, de prendre du recul nécessaire que je n'avais pas forcément toute seule puis de modifier et adapter les façons de procéder. Y a eu le séminaire Changer, les échanges entre nous, entre conseillers, retours des différentes expériences, et fiches de l'Agroseil sur lesquelles je m'appuie maintenant pour préparer débriefer, et puis les visites de terrain qu'on a fait un peu décalées, la visite des cassis [mise en milieu 1 du dispositif] et encore celle de ce séminaire-là [la visite chez l'agriculteur en agriculture biologique, mise en milieu 2 dans le dispositif]

Une autre conseillère rapporte au collectif lors d'un séminaire, une situation qu'elle a vécue avec différents acteurs : agriculteurs, agence de l'eau et collectivité, Direction Départementale Territoriale (DDT). Cette conseillère a pour mission d'accompagner ces différents acteurs dans une bonne gestion et une bonne entente sur la qualité de l'eau en rendant compte de l'évolution des particules chimiques trouvées dans l'eau et partager les évolutions de pratiques et problématiques des agriculteurs. Habituellement ses interventions se font de façon "magistrale " pour présenter les résultats devant les différents acteurs assis face à elle. Dans ce cadre-là, peu d'échanges ont lieu alors qu'ils sont, selon elle, primordiaux pour accompagner des évolutions de pratiques agricoles. Après avoir vécu le tour de plaine dans les cassis, elle décide non pas d'être dans une posture descendante mais de favoriser les échanges des différents acteurs. Pour cela, elle reconfigure les situations habituelles de rencontre entre ces différents acteurs. Ainsi, elle met en œuvre une situation où les différents acteurs seront placés en rond pour que tous puissent se voir pour ainsi réaliser des temps d'échange en sous-groupes. Dans ces sous-groupes, elle choisit de mélanger les différents types d'acteurs pour les amener à confronter les avis et propositions de chacun au regard de ses propres objectifs et ainsi essayer de trouver un consensus. L'activité mise en récit lors de son débriefing de cette situation rapportée par la conseillère au sein du dispositif, met en lumière la capacité de la conseillère à réorienter l'action en cours au regard des indices prélevés en situation en fonction de son but. sur la transition agro-écologique. Après avoir réalisé un tour de plaine dans des 
parcelles pour observer comment se comporte au champ une association de plantes et couverts végétaux (levier agronomique permettant de réduire les risques phytosanitaires et donc de réduire les traitements), le conseiller propose une situation collective nouvelle : une réflexion en salle autour de la construction d'un système de culture sans glyphosate. Pour ces agriculteurs qui fondent leur système sur le nonlabour ${ }^{10}$ et sur l'usage de couverts qu'il faut parfois détruire, cette molécule reste clé. L'invitation est donc de se projeter, d'imaginer un tel système sans nécessairement réinstaurer du labour. Le conseiller est intervenu pour guider les échanges et solliciter l'expérience d'agriculteurs dont il savait qu'ils pouvaient apporter un éclairage sur la problématique. Le système de culture construit après deux heures d'atelier de coconception entre agriculteurs, est une rotation sur 10 ans conjuguant plusieurs leviers agronomiques. Ce travail a permis aux agriculteurs d'identifier ce qu'ils pouvaient faire "dans l'idéal» et les leviers que chacun pouvait remobiliser dans leurs propres pratiques et réflexions sur leurs systèmes de culture. Le conseiller a mis en place les conditions pour que cette situation permette aux agriculteurs de faire émerger leurs raisonnements agronomiques. Son questionnement agronomique lui a permis d'aider les agriculteurs à exprimer leurs règles de décision et à les affiner. Pour un agriculteur cela vient conforter son souhait d'associer «les techniques d'agriculture sous couverts et d'aller en agriculture biologique " et pour un autre de "redécouvrir le métier d'agriculteur ". Pour ce conseiller, s'appuyer sur les expériences des agriculteurs lui permet de favoriser les échanges entre eux, de les amener à être en réflexion sur ce qu'ils font et donc à développer à leur tour leur capacité réflexive pour agir en situation. Cela lui permet de développer une agilité en situation avec ses différents groupes d'agriculteurs en convoquant les expériences de chacun et les agrégeant entre elles pour les amener « au bon moment».

Ces trois conseillers se sont approprié les dimensions agissantes de leurs situations de travail quotidiennes pour les manipuler au regard de leur contexte d'activité respectif. En situation, ils ont aussi montré une réflexivité leur permettant de repérer des événements réorientant leur action, créant ainsi en situation, les ajustements utiles pour développer le potentiel d'apprentissage et de développement de la situation. Inspirés par les deux mises en milieux vécues dans le dispositif, ils développent une capacité à créer des dynamiques collectives d'apprentissage entre acteurs (agriculteurs ou agriculteurs et autres acteurs du territoire) pour leur permettre de coproduire ensemble des connaissances et donner des pistes d'action à chacun.

\section{Discussion-conclusion}

Notre observation longitudinale nous a permis de saisir à la fois ce qui se passe dans le collectif autour de deux situations perturbées créées par les C-A à un an d'intervalle, et d'étudier le développement professionnel qui s'opère via la saisie par les conseillers du potentiel de développement de ces situations pour reconfigurer leur activité et/ou leurs propres situations de travail. Dans l'analyse nous avons distingué deux espaces au sein desquels les conseillers explorent le potentiel de développement de ces situations : l'espace des séminaires organisés par les C-A au sein duquel nous pointons la façon dont, collectivement, les conseillers s'approprient le potentiel de développement des situations qu'ils ont vécues comme perturbantes. L'espace des situations de conseil réelles, avec les agriculteurs, au sein duquel nous mettons en lumière la façon dont des 
conseillers reconfigurent certaines de leurs situations et agissent dans ces situations. Cela nous permet de mettre en exergue que le potentiel de développement des situations créées par les C-A est diversement saisi par les conseillers, et ainsi de pointer la façon dont s'articulent les deux espaces.

L'analyse de ce qui est dit dans le collectif à l'occasion des deux mises en milieu proposées par les C-A, montre que ces situations sont bien plus qu'une "opportunité favorable " (Mayen, 1999) que les conseillers pourraient rencontrer dans leurs propres situations sociales de travail. Les C-A créent une « dynamique des situations » élément primordial, nous disent Pastré et al. (2006), pour que les professionnels puissent "s'ouvrir » aux situations décontextualisées ainsi créées. En perturbant des dimensions prises comme " allant de soi » les C-A les rendent agissantes pour les raisonnements et en font une situation potentielle de développement au sein du dispositif. Cela conduit les conseillers à prendre conscience de leurs façons d'observer une parcelle et de questionner l'agriculteur, principes d'action au cœur du tour de plaine. Si perturber ces dimensions permet une prise de conscience et une ouverture sur d'autres possibles, créer les conditions pour que les conseillers puissent discuter de ce qu'ils ont mis en œuvre pour y faire face renforce le potentiel de développement. Les C-A ont ainsi mis en place des conditions apprenantes via les consignes, les mises en activité selon des conditions singulières et le débriefing. Ce dernier, réalisé collectivement, permet en particulier la reconstruction des significations par l'analyse de l'activité. Ces temps de débriefing collectif, préparé et organisé, permettent aux conseillers d'être des praticiens réflexifs (au sens de Schön, 1994) sur ce qu'ils ont vécu et d'envisager la façon dont cela pourrait faire sens dans leur activité avec leurs agriculteurs. Ainsi, à différents degrés, les conseillers ont acquis des capacités d'analyse réflexive de leurs propres situations de travail et apprennent à analyser leurs dimensions agissantes.

Néanmoins, cette réflexivité n'est pas nécessairement génératrice d'une évolution dans les façons de faire ou de penser. Quand certains conseillers considèrent que «ce n'est pas ce qu'attendent d'eux les agriculteurs", d'autres se construisent des espaces pour expérimenter et dans lesquels ils se sentent capables "d'oser faire autrement ». Nous avons constaté que ces expérimentations sont menées généralement dans des situations où les conseillers ont exprimé, lors des regroupements collectifs, qu'ils n'arrivaient pas à atteindre les objectifs attendus par leurs mandants ou qu'ils se donnaient eux-mêmes. C'est en particulier le cas lorsqu'il s'agit pour eux d'accompagner des agriculteurs dans leurs transitions vers des pratiques plus respectueuses de l'environnement. Les situations perturbées créées par les C-A, en mettant en avant les enjeux du diagnostic et du raisonnement agronomique, ont été leur base de réflexion pour identifier des situations dans lesquelles ils ont osé faire autrement et repérer leurs marges de manœuvre.

Les perturbations créées par les C-A permettent de mettre en suspens le couplage opéré habituellement entre le conseiller et une situation de tour de plaine. Cette mise en suspens rend visible d'autres couplages possibles (de Bisschop, 2020). C'est en travaillant sur ces autres possibles que les conseillers développent, dans leurs propres contextes de travail, une capacité à concevoir des situations potentielles de développement pour leurs collectifs sur le terrain ou encore une capacité d'enquête en situation réelle pour rendre les situations de conseil apprenantes pour les agriculteurs. Cependant nous constatons que la capacité des conseillers à désingulariser (et se désingulariser) une situation est variée. Certains conseillers ont du mal à jouer avec 
certaines dimensions des situations voire ne s'autorisent à manipuler les dimensions des situations que dans certains contextes (par exemple uniquement pour les rencontres avec les agriculteurs dans le cadre d'un territoire BAC). En revanche, d'autres s'autorisent à manipuler les dimensions agissantes des situations et modifient les buts poursuivis jusqu'ici. Cela traduit une évolution de leur activité dans ces situations et de la conceptualisation qu'ils en font. Ces conseillers développent ainsi une capacité renouvelée à tirer profit de nouveaux indices prélevés dans les situations pour maintenir, ou à réorienter si besoin, le cours de l'action en commun de façon à atteindre les buts visés. S'il y a développement professionnel pour l'ensemble des conseillers à travers la prise de conscience de nouveaux possibles, l'actualisation dans l'action de ces possibles est différenciée.

53 Ainsi, la saisie du potentiel de développement des situations créées par les C-A dépend de la façon dont chacun formule les problématiques d'évolution de sa propre façon d'exercer dans certaines situations de conseil. Ce qui est dit dans les temps collectifs permet de considérer que cette saisie dépend autant de la façon dont le conseiller perçoit la situation d'origine comme étant ou non problématique, que des marges de manœuvre qu'il se sent autorisé d'investir tant vis-à-vis de son organisation que des agriculteurs. Certains considèrent qu'ils ne sont pas attendus, tant par les agriculteurs que par leurs mandants sur une nouvelle façon de délivrer un conseil, et ce faisant n'explorent pas les possibles mis en exergue lors des séminaires collectifs. D'autres, qui se sentent confrontés à de nouvelles situations, expriment qu'elles exigent de s'y prendre autrement, et problématisent ce changement au niveau de la technique d'animation qu'ils transposent à partir de ce qu'ils ont vécu dans les mises en milieu. D'autres perçoivent la nécessité de repenser les buts de leur action autant que la façon de s'y prendre. Pour ces conseillers, il s'agit de produire une réflexivité chez les agriculteurs sur leur représentation du champ cultivé. Ils construisent des situations qui viennent changer le rapport que l'agriculteur a à son objet de travail et par ricochet le rapport que l'agriculteur a au conseiller en faisant émerger un raisonnement agronomique via un questionnement agronomique. Enfin, certains vont jusqu'à percevoir les ressorts des mises en milieu qui leur ont été proposées. Ils manipulent les dimensions agissantes des situations en s'attachant à produire eux-mêmes des mises en milieu qui les conduisent à reconsidérer le sens de leur activité. Ce faisant, ils modifient la relation de service qu'ils construisent avec les agriculteurs pour permettre à ces derniers d'élaborer des pratiques agricoles plus respectueuses de l'environnement. Dit autrement, l'appropriation d'une même situation potentielle de développement conçue par des «formateurs " peut aller de la «simple » transposition d'un outil d'animation vécue dans la situation créée par les formateurs, à une reconfiguration des buts et de modes de conduite de l'action dans des situations réelles de travail. Ce gradient dépend des compétences d'enquête développées sur les dimensions agissantes de ces situations, de façon à ajuster l'action pour tenir l'intention visée de « tenir conseil ».

54 Ainsi, notre travail ouvre-t-il à nouveau la piste de situations de travail à didactiser, si le but visé est d'aider une diversité de professionnels à envisager par eux-mêmes comment faire face aux injonctions qui leur sont adressées (ici l'intégration des questions environnementales dans le conseil en production végétale). Et ceci, sans que les situations sociales de conseil ou les activités attendues dans ces situations soient partagées et stabilisées. Cette absence de pratique sociale de référence (Martinand, 1989) complexifie le travail de didactisation et génère de l'incertitude quant à la façon dont les professionnels vont parvenir à développer de nouvelles conceptualisations de 
leurs situations et apprendre à les reconfigurer pour atteindre les buts attendus. L'échange entre pairs fondé sur l'analyse réflexive du couplage entre le professionnel et la situation de travail, s'il permet aux professionnels de mettre en débat le métier (Robert-Guillot, 2015) ne permet pas nécessairement à l'ensemble des professionnels d'entrer dans un mouvement de développement professionnel passant par les trois niveaux de développement évoqués par Pastré et al (2006): réflexivité, désingularisation et reconfiguration par une "réorganisation de ses ressources" lors d'une confrontation à une situation nouvelle. Il ne semble pas non plus suffisant d'aider les conseillers à se saisir de la diversité de leurs situations et problématiques pour distinguer ce qu'il paraît pertinent d'y faire ainsi que le montre notre analyse plus globale du dispositif étudié (Duhamel, 2019). Au vu de ce qui est rapporté par les conseillers qui ne font pas évoluer leur pratique, nous suggérons que c'est dans la construction sociale du dispositif, c'est-à-dire son inscription dans les organisations et dans la façon dont celles-ci structurent la relation de service et définissent les mandats donnés aux conseillers qu'il faut sans doute envisager de travailler dans le futur.

\section{BIBLIOGRAPHIE}

Barbier, J-M., Berton, F., \& Boru, J-J. (1995). Situation de travail, situation de formation. Paris : L'Harmattan.

Bisschop (de), H. (2020). Se former à diriger en situations de formations simulées de haute intensité : une activité de présence à soi et à autrui : une approche par l'activité. Thèse de doctorat, Université Bourgogne Franche-Comté, http://www.theses.fr/2020UBFCH011

Cerf, M., \& Falzon, P. (Eds.). (2005). Situations de service : Travailler dans l'interaction. Paris : Presses Universitaires de France, France.

Cerf, M., \& Maxime, F. (2006). La coproduction du conseil : Un apprentissage difficile. INRA-Educagri, France.

Cerf, M., Omon, B., Guillot, M.-N., Olry, P., \& Petit, M.-S. (2013). Guide «Agroseil »-Vademecum pour échanger sur le métier de conseiller ou animateur en agronomie, RMT Systèmes de culture innovants. Chambre Régionale d'Agriculture Bourgogne Franche-Comté.

Chantre, E. (2011). Apprentissages des agriculteurs vers la réduction d'intrants en grandes cultures : Cas de la Champagne Berrichonne dans les années 1985-2010. Thèse de doctorat, AgroParisTech https:// tel.archives-ouvertes.fr/tel-00675226/

Chizallet, M., Prost, L., \& Barcellini, F. (2020). Supporting the design activity of farmers in transition to agroecology: Towards an understanding. Le Travail Humain, 1(1), 33-59. https:// doi.org/10.3917/th.831.0033

Coquil, X. (2014). Transition des systèmes de polyculture élevage laitiers vers l'autonomie Une approche par le développement des mondes professionnels. Thèse de doctorat, AgroParisTech http:// www.theses.fr/2014AGPT0005 
Coutarel, F., Caroly, S., Vézina, N., \& Daniellou, F. (2015). Marge de manœuvre situationnelle et pouvoir d'agir : des concepts à l'intervention ergonomique. Le Travail Humain, 78(1), 9-29. https:// doi.org/10.3917/th.781.0009

Delbos, C., David, O., Minas, A., Cerf, M., Falgas, C., Gagneur, C.-A., Gilet, J. D., Laudinot, V., Sigwalt, A., \& Waldmeier, E. (2014). Conseil agronomique et réduction des pesticides : Quelles ressources pour affronter ce nouveau challenge professionnel ? Revue AES, 34, 367-378.

Dewey, J. (1967). Logique. La théorie de l'enquête. Paris : PUF.

Duhamel, S. (2019). Les effets développementaux du couplage entre activités et situations de conseil : Analyse d'un dispositif d'échange entre conseillers agricoles accompagnant la transition agro-écologique des agriculteurs. Thèse de doctorat, AgroParisTech, http://www.theses.fr/244428220

Duhamel, S., Cerf, M., \& Olry, P. (2016). Le Tour De Plaine Décalé : Sa conception, sa mise en œuvre et son intérêt pour apprendre, entre conseillers agricoles, le questionnement agronomique. Colloque national « Systèmes de culture innovants : concevoir, former, accompagner », Paris, France.

Duhamel, S., Cerf, M., \& Olry, P. (2017). Analyser son travail et faire face à des situations complexes. Le cas des conseillers agricoles. Education Permanente - Hors-Série, 91-99

Gagneur, C.-A. (2010). Rencontres et interactions au fil du travail, sources de développement. Thèse de doctorat, Université de Bourgogne / Agrosup Dijon, http://www.theses.fr/s12845

Gagneur, C.-A. (2011). Modelisation relation conseil. Rapport Projet CasDAR 9068 Conseiller Demain. Gagneur, C.-A., \& Mayen, P. (2010). Le territoire est-il une situation de développement ? Education Permanente, 184, 63-77.

Guillot, M.-N., Cerf, M., Petit, M.-S., Olry, P., \& Omon, B. (2013). Développer la capacité des conseillers à agir face à la diversité des situations de conseil en grande culture. Économie rurale, 337(5), 59-74.

Lemery, B. (2003). Les évolutions du métier d'agriculteur : Quelles conséquences pour l'appui aux projets des exploitations. Evolution du conseil en agriculture et les métiers du développement. Actes du séminaire sur l'évolution du conseil en agriculture et les métiers du développement, Guyancourt, France, hal-02762678

Martinand, J-L. (1989). Pratiques de référence, transposition didactique et savoirs professionnels en sciences techniques. Les sciences de l'éducation, pour l'ère nouvelle, (2), 23-29.

Mayen, P. (1999). Des situations potentielles de développement. Éducation permanente, 139, 65-86.

Mayen, P. (2007). Quelques repères pour analyser les situations dans lesquelles le travail consiste à agir pour et avec un autre. Recherches en éducation, $n^{\circ} 4,105$.

Mayen, P. (2010). Apprendre du travail collectif à partir de la théorie de l'élaboration de l'action chez Alain Savoyant. Travail et Apprentissages n 5, 55-72.

Mayen, P. (2012). Les situations professionnelles : Un point de vue de didactique professionnelle. Phronesis, 1(1), 59-67.

Mayen, P. (2013). Dinâmica dos saberes e processo de elaboraçao pragmática (Dynamique des savoirs et processus d'élaboration pragmatique). Trabalho \& Educação, 22(3), 125-147.

Mayen, P. (2014). Apprendre à travailler et à penser avec les êtres vivants : L'entrée par la didactique professionnelle. In P. Mayen \& A. Lainé (Eds.), Apprendre à travailler avec le vivant : Développement durable et didactique professionnelle. Éditions Raisons et passions, France. 
Mayen, P., Métral, J.-F., \& Tourmen, C. (2010). Les situations de travail : Références pour les référentiels. Recherche \& formation, 64, 31-46. https://doi.org/10.4000/rechercheformation.191

Mayen, P., \& Olry, P. (2012). Expérience du travail et développement pour de jeunes adultes en formation professionnelle. Recherche \& formation, 70, 91-106. https://doi.org/10.4000/ rechercheformation. 1872

Mayen, P., \& Thiévenaz, J. (2018). Concepts pour une éducation impermanente. Cahiers de l'Herne : Jullien, 158-162

Olivier de Sardan, J-P. (1995). La politique du terrain. Enquête, [En ligne], 1, URL : http:// journals.openedition.org/enquete/263

Omon, B., Cerf, M., Auricoste, C., Olry, P., Petit, M.-S., \& Duhamel, S. (2019). CHANGER - Échanger entre conseillers sur les situations de travail pour accompagner les agriculteurs dans leurs transitions vers l'agroécologie. Innovations Agronomiques, 71, 367-383, DOI : 10.15454/RZKXFZ

Pastré, P. (2011). La didactique professionnelle: Approche anthropologique du développement chez les adultes. Paris : Presses Universitaires de France, France.

Pastré, P., Mayen, P., \& Vergnaud, G. (2006). La didactique professionnelle. Revue française de pédagogie, 1, 1-12.

Prost, L., Berthet, E.T.A., Cerf, M., Jeuffroy, M.-H., Labatut, J., \& Meynard, J.-M. (2017). In-novative design for agriculture in the move towards sustainability: scientific challenges. Research in Engineering Design, Springer Verlag, 28(1), 119-129. DOI : 10.1007/s00163-016-0233-4

Robert-Guillot, M.-N. (2015). Apprendre, tenir et reprendre le métier : Conseiller les agriculteurs en grandes cultures. Thèse de doctorat, Université de Bourgogne, http://www.theses.fr/2015DIJOL013

Vygotski, L.S. (1985). Pensée et langage. Paris : La Dispute.

Vygotski, L. (1988). Los problemas fundamentales de la defectología contemporánea. Cultura y Educación, 11-12, 9-34. ISSN-e 1578-4118

Uwamariya, A., \& Mukamurera, J. (2005). Le concept de développement professionnel en enseignement : approches théoriques. Revue des sciences de l'éducation, 31(1), 133-155.

\section{NOTES}

1. L'AFEST est un dispositif d'«Actions de Formation En Situation de Travail» créé depuis septembre 2018.

2. Nous utilisons le masculin dans le texte bien que cette forme recouvre l'ensemble de la population féminine qui exerce un métier.

3. Le tour de plaine est une situation sociale de conseil récurrente en production végétale. Il se réalise entre février et avril-mai généralement. Le conseiller va dans une parcelle cultivée avec un ou des agriculteurs, pour observer l'état du champ à cette période de l'année, identifier des problèmes qui peuvent nécessiter une action immédiate de l'agriculteur comme apporter de l'azote, traiter contre une maladie, désherber le champ. Le conseiller questionne sur les pratiques mises en place jusqu'ici par l'agriculteur sur cette parcelle, fait un diagnostic du problème avec lui, et propose une solution et souvent rappelle les règles de décision permettant de prendre les décisions et les indicateurs (par exemple : seuil d'infestation, stade de la culture, etc.) pour porter le diagnostic.

4. Cette technique, issue de l'ethnographie et de l'anthropologie, consiste à mener une analyse tout en étant intégré au processus de production, ce qui permet de développer une certaine 
«familiarisation » avec le milieu et d'accéder à des informations au plus proche des faits. Dans le cas présent, certaine participation à la construction de l'animation et à la réflexivité des C-A a également eu lieu de la part du premier auteur.

5. Le système de culture est l'espace sur lequel s'applique une succession identique de cultures et d'opérations techniques réalisées de façon cohérente sur chacune de ces cultures en lien avec les objectifs de conduite assignées à celles-ci.

6. L'itinéraire technique est la suite ordonnée et cohérente des actions réalisées sur une culture et une parcelle donnée en vue d'objectifs à atteindre.

7. Les adventices sont «les mauvaises herbes» soit des plantes qui poussent dans une parcelle sans y avoir été intentionnellement installée.

8. Le Ray Grass est une plante graminée considérée comme une adventice dans les champs de céréales cultivés par les agriculteurs mais pouvant être cultivée comme fourrage par d'autres.

9. Le vulpin est une plante graminée considérée comme adventice par les agriculteurs.

10. Le labour est un élément clé en agriculture biologique par exemple pour gérer les adventices et contrôler le stock de graines dans le sol. Les agriculteurs qui renoncent au labour le font à la fois pour des raisons de gain de temps et de fioul, mais aussi pour avoir " un sol vivant » en limitant les perturbations physiques liées au labour qui modifient l'environnement des organismes vivants (vers de terre, microfaune et flore du sol).

\section{RÉSUMÉS}

Les professionnels qui agissent avec et pour autrui, dont les activités et situations évoluent et ne sont pas stabilisées, ont pour enjeu de reconstruire avec leurs bénéficiaires le sens à donner à leurs activités et aux situations de travail conjointes. C'est le cas des conseillers agricoles mis en situation d'accompagner des agriculteurs dans une évolution de leurs pratiques pour répondre à des problématiques environnementales. Un dispositif d'échange entre pairs a été mis en place auprès de ces professionnels, réunis pour penser collectivement ce qui rend leur action efficace aujourd'hui et pour tenter d'autres pratiques, pour oser faire autrement demain. Nous avons réalisé une observation participante au sein de ce dispositif. Nous étudions plus particulièrement la façon dont, collectivement et individuellement, les participants se saisissent de deux «mises en milieu » conçues par les animatrices du dispositif autour d'une situation emblématique du conseil en production végétale : le tour de plaine. Nos descriptions et analyses montrent la façon différenciée dont les conseillers s'approprient le potentiel de développement des situations ainsi créées pour changer leur activité en situation réelle de conseil. Nous discutons du développement professionnel que cela traduit et pointons des pistes d'améliorations possibles de l'animation pour mieux intégrer la diversité des participants et de leur environnement de travail.

For professionals who work with and for others and whose activities and situations evolve and are not yet stabilized, one of the challenges is that of reconstructing, with their beneficiaries, the purpose of their activities and of reconfiguring their work situations. This is the case for agricultural advisors who are in the position of accompanying farmers in a change of practices in order to cope with environmental problems. A system was designed to support exchanges between advisors so that they can collectively rethink the efficacy of their action, and dare to act differently. We conducted a participatory observation within this system. We studied the way in which, individually and collectively, the participants grasp the two "milieus" designed by the 
facilitators in relation to an emblematic advisory situation for crop production: the field tour. Our illustrations and analyses show the different ways that the advisors grasp the developmental potential of these designed situations in order to change their own activity in real work situations. We discuss the professional development that this creates. Finally, we highlight possible avenues for improving the facilitation work to better cope with the diversity of the participants and of their workplace.

\section{INDEX}

Mots-clés : échanges entre pairs, situation potentielle de développement, conseil agricole, agroécologie

Keywords : peer exchanges, situation of potential development, agricultural advice, agroecology

\section{AUTEURS}

\section{SOPHIE DUHAMEL}

LISIS, Univ Gustave Eiffel, ESIEE Paris, CNRS, INRAE, F-77454 Marne-la-Vallée, France, Université BFC, AgroSupDijon, BP87999, 21079 Dijon Cedex, sof.duhamel@gmail.com

\section{PAUL OLRY}

Université BFC, AgroSupDijon, BP87999, 21079 Dijon Cedex, paul.olry@agrosupdijon.fr

\section{MARIANNE CERF}

Université Paris-Saclay, INRAE, AgroParisTech, UMR SAD-APT, 75005 Paris, France, marianne.cerf@inrae.fr 